\title{
AVALIAÇÃO DA PRODUÇÃO DOS COMPOSTOS MAJORITÁRIOS DA FERMENTAÇÃO DE MOSTO DE UVA POR LEVEDURAS ISOLADAS DA REGIÃO DA "SERRA GAÚCHA" (RS)"
}

\author{
Maria Eugênia de Oliveira MAMEDE ${ }^{2, *}$, Gláucia Maria PASTORE ${ }^{2}$
}

\begin{abstract}
RESUMO
O objetivo deste trabalho foi estudar o comportamento do crescimento, bem como a produção de compostos voláteis durante a fermentação de mosto de uva pelas leveduras Kloeckera apiculata e Saccharomyces cerevisiae. As concentrações dos compostos voláteis majoritários da fermentação foram dependentes da temperatura de fermentação. Nas fermentações a $20^{\circ} \mathrm{C}$, as concentrações da massa celular seca e dos compostos voláteis foram maiores do que na fermentação a $15^{\circ} \mathrm{C}$. A Kloeckera apiculata produziu altas concentrações de acetato de etila $(197,0 \mathrm{mg} / \mathrm{L}-310,0 \mathrm{mg} / \mathrm{L})$ e acetato de isoamila $(19,3 \mathrm{mg} / \mathrm{L}-31,3 \mathrm{mg} / \mathrm{L})$, ésteres de grande importância sensorial. No entanto, a concentração de etanol obtida foi baixa, cerca de 6,3g/L - 24,0g/L, em comparação à conseguida utilizando Saccharomyces cerevisiae como agente de fermentação $(27,3 \mathrm{~g} / \mathrm{L}-34,0 \mathrm{~g} / \mathrm{L})$

Palavras-chave: fermentação; vinho; produção de aroma; isolamento; Kloeckera apiculata; Saccharomyces cerevisiae.
\end{abstract}

\section{SUMMARY}

EVALUATION OF THE MAJOR COMPOUNDS FORMED DURING GRAPE MUST FERMENTATION BY YEAST ISOLATED FROM “SERRA GAÚCHA” (RS) REGION. The objective of this work was to study the growth behavior, as well as, volatile compounds production during grape must fermentation by Kloeckera apiculata and Saccharomyces cerevisiae. The concentrations of the major volatile compounds during the fermentation were dependent of the temperature of the fermentation. In fermentation at $20^{\circ} \mathrm{C}$, the concentration of the dry weight biomass and volatile compounds were greater than that ones at $15^{\circ} \mathrm{C}$. The Kloeckera apiculata produced high concentrations of the ethyl acetate $(197.0 \mathrm{mg} / \mathrm{L}-310.0 \mathrm{mg} / \mathrm{L})$ and isoamyl acetate $(19.3 \mathrm{mg} / \mathrm{L}-31.3 \mathrm{mg} / \mathrm{L})$, esters of great sensory importance. However, the ethanol concentration obtained was lower, about $6.3 \mathrm{~g} / \mathrm{L}-24.0 \mathrm{~g} / \mathrm{L}$, than that one obtained with fermentation by Saccharomyces cerevisiae $(27.3 \mathrm{~g} / \mathrm{L}-34.0 \mathrm{~g} / \mathrm{L})$.

Keywords: fermentation; wine; aroma production; isolated; Kloeckera apiculata; Saccharomyces cerevisiae.

\section{1 - INTRODUÇÃO}

O processo tradicional de obtenção do vinho, fermentação natural (espontânea) do mosto de uva é realizada por uma seqüência de diferentes espécies de leveduras. Inúmeras leveduras, que não-Saccharomyces cerevisiae, como as do gênero Saccharomyces, Kloeckera, Candida, Hansenula, Hanseniospora, Pichia, Zigossacharomyces, entre outras, fazem parte da microflora da uva e podem iniciar a fermentação [11].

O crescimento destas leveduras durante a fermentação é influenciado por diferentes fatores como $\mathrm{pH}$, concentração de nitrogênio e açúcar, concentração de $\mathrm{SO}_{2}$, conteúdo de álcool e ainda pela temperatura de fermentação [12]. As leveduras do gênero Saccharomyces são resistentes a altas concentrações de etanol e por isso dominam a fermentação alcoólica de mosto de uva para produção de vinho. Já as leveduras do genêro Kloeckera, Candida, Hanseniospora, Hansenula, Pichia e Kluyveromyces, comuns a microflora da uva, são menos resitentes ao etanol e morrem no terceiro ou quarto

\footnotetext{
1. Recebido para publicação em 26/08/2003. Aceito para publicação em 14/05/2004 (001206).

2. Laboratório de Bioaromas - Departamento de Ciência de Alimentos. Faculdade de Engenharia de Alimentos - Universidade Estadual de Campinas R: Monteiro Lobato, 80. C.P. 6121, CEP: 13083-970. Cidade Universitária, Campinas - S.P. Fone: (19) 37882175. E-mail: mamedema@bol.com.br

* A quem a correspondência deve ser enviada.
}

dia de fermentação [9]. Embora estas leveduras participem apenas do início da fermentação, elas produzem compostos de aroma que têm grande contribuição para a qualidade sensorial do vinho. Por isso, o estudo das propriedades metabólicas e fisiológicas das leveduras não-Saccharomyces cerevisiae pode ser importante para a indústria de vinhos. A Kloeckera apiculata tem capacidade de produzir altas concentrações de compostos voláteis tais como: álcoois superiores, ésteres e ácidos, compostos de carbono conhecidos como produtos secundários da fermentação $[10,18,19]$.

Este trabalho procurou investigar a produção de compostos de aroma pelas leveduras Kloeckera apiculata e Saccharomyces cerevisiae durante a fermentação alcoólica dos mostos de uvas Chardonnay e Pinot Noir sob temperaturas de $15^{\circ} \mathrm{C}$ e $20^{\circ} \mathrm{C}$.

\section{2 - MATERIAL E MÉTODOS}

\section{1 - Leveduras}

As leveduras Saccharomyces cerevisiae e a Kloeckera apiculata foram isoladas do bago de uva Chardonnay cultivada na região da Serra Gaúcha (RS). As várias colônias isoladas em placa de Petri contendo meio "yeast malt" ( $1 \%$ de glicose; $2 \%$ de ágar; 0,5\% de peptona; $0,3 \%$ malte e $0,3 \%$ de extrato de levedura) foram selecionadas com base em sua morfologia e repicadas novamente. Análises microscópicas verificaram a pureza do isolado. $\mathrm{O}$ isolado foi mantido em meio descrito acima com sucessivos repiques de 15 em 15 dias até a sua identificação. 


\section{2 - Identificação das leveduras}

A identificação das duas leveduras foi realizada pela galeria ID 32 C (Biomeriux) segundo PINA \& HOGG [15]. Esta galeria é padronizada para identificação de leveduras que utiliza mini-testes de assimilação padronizados e uma base de dados experimentais adaptada. A interpretação da leitura da galeria ID $32 \mathrm{C}$ foi realizada pelo modo automático mini-API (Biomeriux).

\section{3 - Avaliação físico-química}

Os mostos puros Chardonnay e Pinot Noir sofreram a avaliação do conteúdo de açúcar, nitrogênio amoniacal, $\mathrm{SO}_{2}$ livre e total, acidez volátil e total e valor de $\mathrm{pH}$, bem como da estimativa da quantidade de álcool provável de ser formada. Os dados da concentração de açúcar e álcool foram obtidos pela relação com os valores da densidade calculada pelo densímetro Mustimetre DujardinSalleron (385200). O valor de $\mathrm{pH}$ foi medido em $\mathrm{pHmetro}$ (Metter Toledo 320- Tecnal). A concentração de acidez volátil foi calculada pelo destilador Gilbertini (Distillatore Eletrônic Enochimic). O valor da concentração da acidez total foi obtido por titulação de acordo com OUGH \& AMERINE [14]. A quantidade de $\mathrm{SO}_{2}$ livre e total foi obtida por uma adaptação do método de RIBEREAU \& PEYNAUD [16]. A concentração de nitrogênio amoniacal foi obtida pelo método de OUGH [13].

\section{4 - Condições de fermentação dos mostos Chardonnay e Pinot Noir}

Mostos de uvas Chardonnay e Pinot Noir, doados pela indústria Chandon do Brasil S/A, situada no Estado do Rio Grande do Sul (RS), foram usados para investigação da produção de aroma por duas leveduras durante uma fermentação de sete dias. A fermentação foi iniciada após a adição de $1 \times 10^{7}$ células $/ \mathrm{mL}(K$. apiculata ou S. cerevisiae) em erlenmeyers de $125 \mathrm{~mL}$ contendo $25 \mathrm{~mL}$ de mosto. Antes de iniciar a fermentação os mostos foram filtrados em membrana (Millipore) de $0,22 \mu \mathrm{m}$. Os erlenmeyers tampados foram incubados a temperaturas de $20^{\circ} \mathrm{C}$ e $15^{\circ} \mathrm{C}$, temperaturas as quais são as mais próximas das condições de fermentação para produção industrial de vinho. A cada 24 horas, um frasco, contendo $25 \mathrm{~mL}$ de mosto fermentado foi filtrado

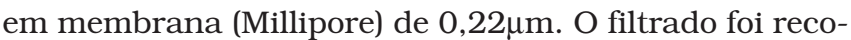
lhido e o seu pH determinado de acordo com o item 2.3. A concentração celular, expressa em massa seca (MS), foi quantitativamente determinada através de secagem destas membranas a $100^{\circ} \mathrm{C}$, por 24 horas, e divisão pelo correspondente volume filtrado. As fermentações foram realizadas em triplicatas.

\section{5 - Condições cromatográficas para análise dos compostos voláteis}

a) Os compostos voláteis foram isolados pelo sistema de "Purge and Trap concentrator/Dynamic Headspace” (G1900-60500), usando o polímero Tenax. As condições de isolamento e concentração dos voláteis foram: Temperatura da amostra: $30^{\circ} \mathrm{C}$; Tempo de purga: 15 minutos; velocidade de fluxo do gás de arraste: $30 \mathrm{~mL} / \mathrm{min}$.; Temperatura de desorção: $180^{\circ} \mathrm{C}$; Tempo de desorção: 10min. A temperatura da linha de transferência usada foi de $180^{\circ} \mathrm{C}$.

b) O cromatógrafo gasoso HP-G 1908-60500 (FID) foi equipado com uma coluna capilar HP-Innowax (30m $\times 0,25 \mathrm{~mm} \times 0,25 \mathrm{~mm})$, de fase ligada polietilenoglicol. O gás de arraste utilizado foi o Hélio: $1 \mathrm{~mL} / \mathrm{min}$. A programação da temperatura da coluna foi: $35^{\circ} \mathrm{C} / 5 \mathrm{~min}$., rampa: $3^{\circ} \mathrm{C} / \mathrm{min} . \rightarrow 140^{\circ} \mathrm{C}$ $\rightarrow 180^{\circ} \mathrm{C} / 5 \mathrm{~min}$., temperatura do detector: $250^{\circ} \mathrm{C}$, temperatura do injetor: $200^{\circ} \mathrm{C}$. O modo de injeção foi do tipo "splitless".

c) As análises de identificação foram realizadas em um cromatógrafo gasoso Shimadzu - (EM) QP 5000 (Espectrometria de massas). A programação da temperatura da coluna foi igual à seqüência reportada acima, com exceção para o sistema de injeção, o qual foi utilizado o modo split $(1: 100)$. A voltagem de ionização aplicada foi de $70 \mathrm{eV}$ e o espectro de massa obtido de uma faixa de varredura de $35-350 \mathrm{~m} / \mathrm{z}$.

A quantificação foi realizada pelo método de padronização externa usando gráficos de padrões de voláteis correspondentes da Sigma-Aldrich e Merck. As análises foram realizadas em triplicatas.

\section{3 - RESULTADOS E DISCUSSÃO}

\section{1 - Seleção das leveduras}

As leveduras foram identificadas de acordo com sistema automático mini-API ID 32 C como Saccharomyces cerevisiae e Kloeckera apiculata. A intervalo de confiança da identificação do gênero dada pelo sistema automático foi de 99,8\% e 99,9\% para a Saccharomyces cerevisiae e Kloeckera apiculata, respectivamente.

\section{2 - Dados físico-químicos dos mostos Chardonnay e Pinot Noir puros (sem fermentação).}

As análises físico-químicas dos mostos foram realizadas com o intuito de verificar se estes estariam com concentrações de açúcar, nitrogênio, acidez, $\mathrm{SO}_{2}$ dentro de limites propícios para iniciar a fermentação. Os dados destas análises estão apresentados na Tabela 1.

TABELA 1. Avaliação físico-química dos mostos.

\begin{tabular}{lcc}
\hline \multicolumn{1}{c}{ Parâmetros Químicos } & Mosto Chardonnay & Mosto Pinot Noir \\
\hline Densidade & $1,074^{1{ }^{\circ{ }^{\circ} \mathrm{C}}}$ & $1,071^{1{ }^{\circ{ }^{\circ}}}$ \\
\% de álcool provável & 10,23 & 8,91 \\
Açúcar (g/L) & 172,10 & 164,40 \\
Acidez total (g/L) & 4,90 & 3,70 \\
$\mathrm{SO}_{2}$ livre (mg/L) & 5,60 & 9,90 \\
$\mathrm{SO}_{2}$ total (mg/L) & 19,80 & 38,66 \\
$\mathrm{NH}_{4}{ }^{+}$(mg/L) & 76,60 & 75,60 \\
$\mathrm{pH}^{\text {Acidez volátil (g/L) }}$ & 3,20 & 3,21 \\
\hline
\end{tabular}

Média de 3 repetições. 
Segundo BISSON [3] uma fermentação de 25 a 55 dias necessita de uma concentração de açúcar em torno de $150-240$ g/L e uma concentração mínima de nitrogênio da forma amoniacal em torno de 70-140mg/L.

A presença de $\mathrm{SO}_{2}$, bem como sua quantidade, no mosto regula a atividade microbiana e impede o crescimento de alguns microrganismos. A quantidade de 1 a $10 \mathrm{mg} / \mathrm{L}$ de $\mathrm{SO}_{2}$ livre é o suficiente para que não ocorra contaminação do mosto por bactérias lácticas durante a fermentação alcoólica [17].

$\mathrm{O}$ pH do mosto para produção de vinho espumante deve estar em torno de 3 [4]. De acordo com BOULTON et al. [4], BISSON [3] e ROMANO \& SUZZI [17], os mostos utilizados neste trabalho estão em condições propícias para iniciar a fermentação.

\section{3 - Variação da massa celular seca e pH dos mos- tos Chardonnay e Pinot Noir.}

A variação da massa celular durante a fermentação a $20^{\circ} \mathrm{C}$ dos mostos Chardonnay e Pinot Noir pela Kloeckera apiculata esta representada na Figura 1. A fase de crescimento da Kloeckera apiculata no mosto Chardonnay apresentou um rápido crescimento nas 72 horas iniciais da fermentação. A sua massa celular alcançou uma concentração de $12,3 \mathrm{mg} / \mathrm{L}$ (expressa em massa seca) em 168 horas de fermentação. No mosto Pinot Noir o comportamento da variação da massa celular foi muito similar. O período de maior crescimento aconteceu nas 72 horas iniciais da fermentação. Após este período, houve um declínio da velocidade, correspondendo à fase estacionária. Em 168 horas de fermentação, a concentração celular obtida foi de $10,1 \mathrm{mg} / \mathrm{L}$

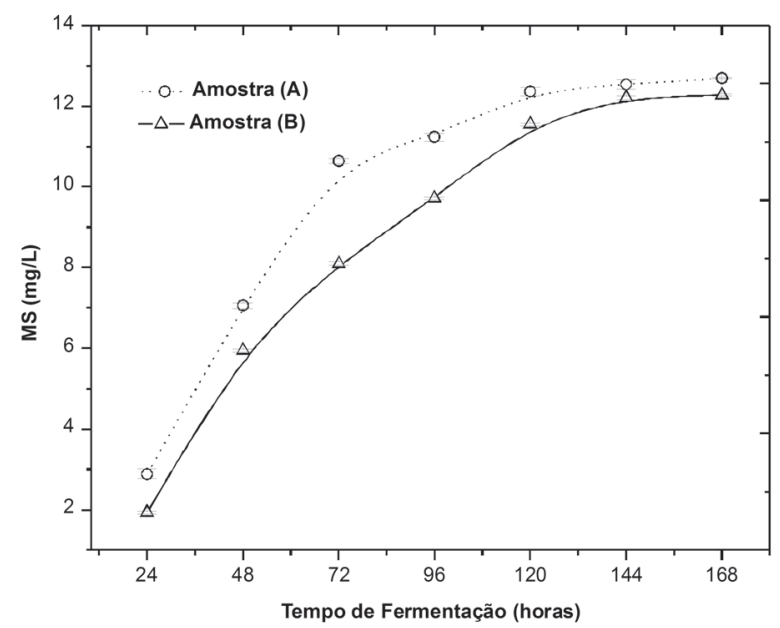

FIGURA 1. Concentração celular, expressa em massa seca (MS) para fermentação por Kloeckera apiculata a $20^{\circ} \mathrm{C}$. (A) Mosto Pinot Noir, (B) Mosto Chardonnay.

A variação da concentração celular nos mostos Chardonnay e Pinot Noir fermentados a $20^{\circ} \mathrm{C}$ com a Saccharomyces cerevisiae está representada na Figura 2. A Saccharomyces cerevisiae mostrou um rápido crescimento nas 72 horas iniciais da fermentação no mosto Chardonnay, mas após este período o crescimento prati- camente estagnou. A concentração celular de $12,4 \mathrm{mg} / \mathrm{L}$ (expressa em massa seca) foi alcançada totalmente nas 72 horas iniciais da fermentação. A fermentação no mosto Pinot Noir pela Saccharomyces cerevisiae mostrou ser rápida e brusca nas 48 horas iniciais da fermentação. Após este período o crescimento foi diminuindo até alcançar a fase estacionária, entre 144 e 168 horas de fermentação.

Os dados reportados acima estão similares aos de FLEET \& HEARD [9], onde estes autores verificaram que as leveduras do gênero Saccharomyces e Kloeckera atingem sua fase de maior crescimento nas 72 horas iniciais da fermentação.

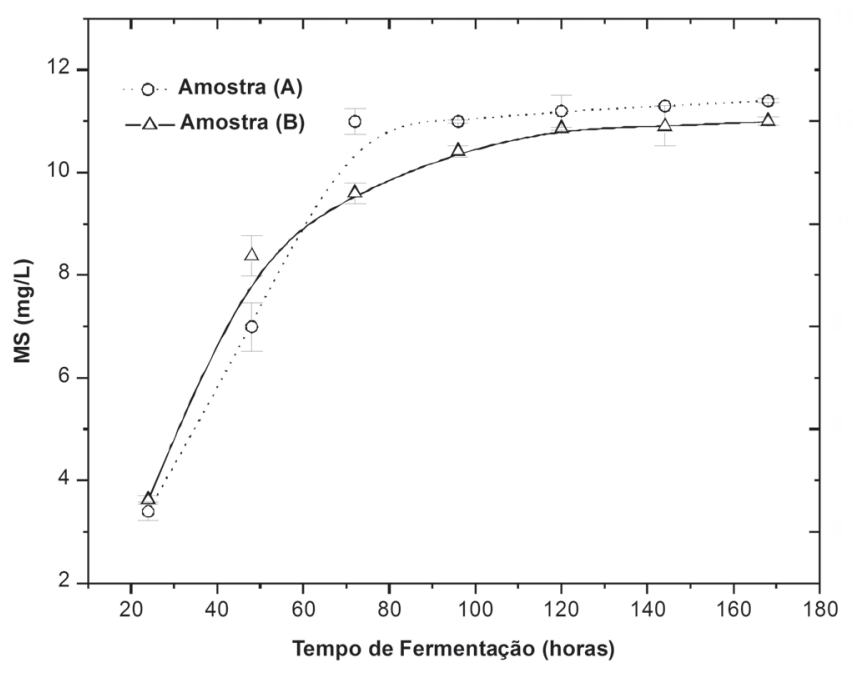

FIGURA 2. Concentração celular, expressa em massa seca (MS) para fermentação por Saccharomyces cerevisiae a $20^{\circ}$ C. (A) Mosto Pinot Noir, (B) Mosto Chardonnay.

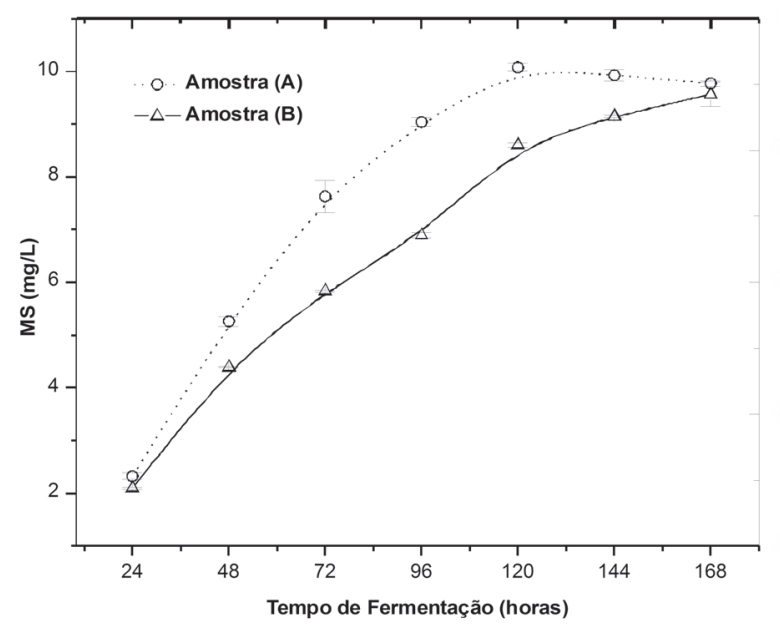

FIGURA 3. Concentração celular, expressa em massa seca (MS) para fermentação por Kloeckera apiculata a $15^{\circ} \mathrm{C}$. (A) Mosto Pinot Noir, (B) Mosto Chardonnay.

O crescimento celular a $15^{\circ} \mathrm{C}$ mostrou ser um pouco diferente daquele a $20^{\circ} \mathrm{C}$. As concentrações celulares das leveduras Saccharomyces cerevisiae e Kloeckera apiculata alcançadas após 168 horas de fermentação a $15^{\circ} \mathrm{C}$ foram menores e as fases exponenciais de cresci- 
mento foram lentas, acontecendo entre 72 e 96 horas de fermentação. Neste caso, a $15^{\circ} \mathrm{C}$, a fermentação mostrou ser um pouco mais lenta. As concentrações celulares máximas da levedura Kloeckera apiculata foram equivalentes tanto no mosto Chardonnay como no mosto Pinot Noir (Figura 3), pois pelo teste de Análise de Variância (ANOVA) não apresentaram diferenças significativas.

As variações das concentrações celulares da Saccharomyces cerevisiae nos mostos Chardonnay e Pinot Noir estão representadas na Figura 4. Esta levedura apresentou sua fase de maior crescimento nas 72 horas iniciais da fermentação tanto no mosto Chardonnay quanto no Pinot Noir. Após as 168 horas de fermentação, a concentração celular foi de $10,4 \mathrm{mg} / \mathrm{L}$ (expressa em massa seca), não apresentando diferenças significativas em relação aos mostos de uvas utilizados.

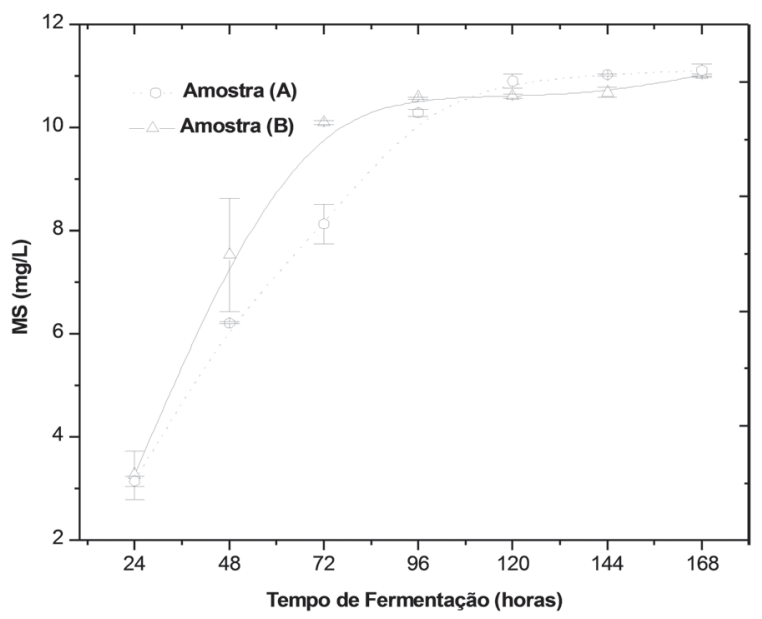

FIGURA 4. Concentração celular, expressa em massa seca (MS) para fermentação por Saccharomyces cerevisiae a $15^{\circ}$ C. (A) Mosto Pinot Noir, (B) Mosto Chardonnay.

Estes dados mostraram que os meios de fermentação (mostos Chardonnay e Pinot Noir), mesmo com concentrações diferentes de açúcar, não tiveram influência nas fases de crescimento, nem mesmo no valor da concentração celular máxima obtida.

$\mathrm{O}$ valor de $\mathrm{pH}$ inicial, que era de 3,20 , durante a fermentação a $20^{\circ} \mathrm{C}$ caiu para 2,80 , enquanto que na fermentação a $15^{\circ} \mathrm{C}$ o $\mathrm{pH}$ final permaneceu em torno de 2,90 .

\section{4 - Compostos voláteis da fermentação}

O isolamento dos voláteis pelo sistema automático "Purge and Trap/Concentrator-Dynamic Headspace" mostrou que a técnica foi muito eficaz para compostos de baixo ponto de ebulição. Segundo FERREIRA et al. [8] esta técnica não é sensível para analisar compostos com ponto de ebulição médio e alto. Por esta técnica, foi possivel isolar 22 compostos de aroma presentes nas amostras após 168 horas de fermentação (Tabela 2).

Não houve diferenças da detecção dos compostos voláteis dos mostos fermentados a $15^{\circ} \mathrm{C}$ e a $20^{\circ} \mathrm{C}$ (Tabela 2).
TABELA 2. Composição de voláteis das amostras de mostos após 168 horas de fermentação tanto a $20^{\circ} \mathrm{C}$ quanto a $15^{\circ} \mathrm{C}$.

\begin{tabular}{lc}
\hline \multicolumn{1}{c}{ Compostos voláteis detectados } & Amostra \\
\hline 1) Etanol & 1 e 2 \\
2) 1-Hexenol & 3 e 4 \\
3) Isobutanol & $1,2,3$ e 4 \\
4) Álcool isoamílico & $1,2,3$ e 4 \\
5) 1-Propanol & $1,2,3$ e 4 \\
6) Butanol & 4 \\
& \\
7) Acetato de etila & $1,2,3$ e 4 \\
8) Acetato de metila & 1 e 2 \\
9) Acetato de propila & 1 \\
10) Acetato de isopropila & 2 \\
11) Acetato de isoamila & $1,2,3$ e 4 \\
12) Propionato de etila & 1 \\
13) Butirato de etila & 3 \\
14) Butil isobutirato & 3 \\
& \\
15) 3-Hidroxi-2-butanona & 2 \\
16) n-propanal & 2,3 e 4 \\
17) 1-Butanal & 2,3 e 4 \\
18) 3-metil, butanal & 2,3 e 4 \\
19) Isobutiraldeído & 2,3 e 4 \\
20) Acetaldeído & 1 e 2 \\
21) Ácido acético & $1,2,3$ e 4 \\
22) Ácido capróico & \\
\hline
\end{tabular}

Amostras: $1=$ Mostos Chardonnay e Pinot Noir fermentados por Kloeckera apiculata; $2=$ Mostos Chardonnay e Pinot Noir fermentados por Saccharomyces cerevisiae; 3 = Mosto Chardonnay sem fermentação; 4 = Mosto Pinot
Compostos identificados por Espectrometria de massas.

TABELA 3. Valores das médias da concentração dos compostos majoritários após 168 horas de fermentação.

\begin{tabular}{|c|c|c|c|c|c|c|c|}
\hline \multirow[b]{2}{*}{ Amostra } & \multicolumn{7}{|c|}{ Médias das Concentrações } \\
\hline & Acetaldeído & Etanol (g/L) & $\begin{array}{l}\text { 1-propanol } \\
\text { (mg/L) }\end{array}$ & $\begin{array}{c}\text { Isobutanol } \\
(\mathrm{mg} / \mathrm{L})\end{array}$ & $\begin{array}{c}\text { Álcool } \\
\text { isoamílico } \\
(\mathrm{mg} / \mathrm{L})\end{array}$ & $\begin{array}{c}\text { Acetato de } \\
\text { etila } \\
(\mathrm{mg} / \mathrm{L})\end{array}$ & $\begin{array}{c}\text { Acetato de } \\
\text { isoamila } \\
(\mathrm{mg} / \mathrm{L})\end{array}$ \\
\hline 1 & $131,3^{\mathrm{a}}$ & $34,0^{2}$ & $9,0^{20}$ & $54,0^{2}$ & $7,0^{\circ}$ & $184,0^{\circ}$ & $20,7^{\circ \mathrm{C}}$ \\
\hline 2 & $125,3^{\mathrm{a}}$ & $32,0^{\mathrm{ab}}$ & $7,3^{6}$ & $52,3^{a}$ & $6,0^{\circ}$ & $194,0^{b}$ & $20,3^{b c}$ \\
\hline 3 & $53,3^{\mathrm{d}}$ & $24,0^{b c}$ & $15,3^{\mathrm{a}}$ & $20,7^{\circ}$ & $112,3^{b}$ & $310,0^{a}$ & $31,3^{a}$ \\
\hline 4 & $45,7^{\mathrm{d}}$ & $22,0^{c d}$ & $11,7^{\mathrm{ab}}$ & 19,0 & $126,0^{2}$ & $295,0^{a}$ & $24,0^{b}$ \\
\hline 1 & $99,0^{b}$ & $28,0^{\mathrm{abc}}$ & $8,0^{\circ}$ & $37,3^{b}$ & $5,0^{\circ}$ & $110,0^{\circ}$ & $15,0^{6}$ \\
\hline $2^{\prime}$ & $80,3^{c}$ & $27,3^{\mathrm{abc}}$ & $6,0^{b}$ & $36,0^{b}$ & $5,0^{d}$ & $120,0^{\circ}$ & $14,7^{\circ}$ \\
\hline $3^{\prime}$ & $15,0^{e}$ & $15,3^{d}$ & $9,7^{\mathrm{ab}}$ & $18,0^{\circ}$ & $98,3^{c}$ & $200,0^{b}$ & $19,7^{\mathrm{bc}}$ \\
\hline $4^{\prime}$ & $14,3^{\mathrm{e}}$ & $6,3^{\mathrm{e}}$ & $9,3^{\text {ab }}$ & $18,3^{6}$ & $98,0^{\circ}$ & $197,0^{\circ}$ & $19,3^{\mathrm{bc}}$ \\
\hline
\end{tabular}

As fermentações das amostras 1-4 foram realizadas a $20^{\circ} \mathrm{C}: 1=$ Mosto Chardonnay fermentado pela Saccharomyces cerevisiae, $2=$ Mosto Pinot Noir fermentado pela Saccharomyces cerevisiae, 3 = Mosto Chardonnay fermentado pela Kloeckera apiculata, $4=$ Mosto Pinot Noir fermentado pela Kloeckera apiculata. As fermentaccões das
amostras de 1' a $4^{\prime}$ ' foram realizadas a $15^{\circ} \mathrm{C}$ e seguem a mesma seqüência de ordem amostras de $1^{\prime}$ a $4^{\prime}$ ' foram realizadas a $15^{\circ} \mathrm{C}$ e seguem a mesma seqüência de ordem tivamente pelo teste de Tukey $(\mathrm{p} \leq 0,05)$.

BILBAO et al. [2] observaram que a produção de ésteres, álcoois superiores e aldeídos durante a fermentação alcoólica pela Saccharomyces cerevisiae e Kloeckera apiculata são influenciados pela temperatura de fermentação. Neste trabalho foi observado que a concentração dos compostos voláteis variou em rela- 
ção às temperaturas de fermentação utilizadas. Após 168 horas de fermentação dos mostos Chardonnay e Pinot Noir pelas leveduras Saccharomyces cerevisiae e Kloeckera apiculata, a concentração dos compostos majoritários da fermentação como o acetaldeído, acetato de etial, etanol, 1-propanol, Isobutanol, acetato de isoamila e álcool isoamílico foram quantificados (Tabela 3). A fermentação a $20^{\circ} \mathrm{C}$ favoreceu a produção de uma maior concentração dos compostos voláteis majoritários de aroma do que a fermentação realizada a $15^{\circ} \mathrm{C}$.

De um modo geral, a produção dos voláteis majoritários da fermentação como o etanol, 1-propanol, álcool isoamílico, isobutanol, acetaldeído, acetato de isoamila, acetato de etila não apresentaram diferenças significativas em relação aos mostos utilizados (Chardonnay ou Pinot Noir). Com exceção das amostras 1' e 2' (mosto Chardonnay e Pinot Noir fermentado com Saccharomyces cerevisiae), onde as concentrações de acetaldeído diferiram estatisticamente. O composto que apresentou as maiores diferenças estatísticas de produção em relação às temperaturas de fermentação foi o acetaldeído. $\mathrm{O}$ acetaldeído é um importante composto da fermentação, correspondendo por $90 \%$ dos aldeídos totais do vinho e sua formação advém da descarboxilação do piruvato [5,7].

Os 3-metil-butanol (álcool isoamílico), 2-metil-butanol (álcool amil ativo), 2-metil-propanol (isobutanol) e 1-propanol (n-propanol) são chamados de álcoois superiores e são oriundos do metabolismo de aminoácidos ou açúcares durante a fermentação alcoólica [1].

Nas amostras de mosto de uva fermentado pela Kloeckera apiculata a $15^{\circ} \mathrm{C}$ foram observadas as mais baixas concentrações de etanol em comparação às demais amostras. FLEET \& HEARD [9] já haviam verificado que a fermentação realizada pela cultura pura de Kloeckera apiculata apresentava baixas quantidades de etanol, cerca de $6,5 \%(v / v)$. A produção de 1-propanol não diferiu estatisticamente em relação às temperaturas de fermentação. A concentração do isobutanol nas amostras de mostos de uvas fermentados com a Saccharomyces cerevisiae a $15^{\circ} \mathrm{C}$ mostrou ser estatisticamente diferente da concentração deste composto obtido a $20^{\circ} \mathrm{C}$. A produção de isobutanol pela Kloeckera apiculata não apresentou diferenças estatísticas em relação à temperatura de fermentação (amostras 3, 4, 3' e 4'). Com relação à produção de álcool isoamílico as amostras que não diferiram significativamente foram 1, 2, 1' e 2' (mostos de uvas fermentados a $20^{\circ} \mathrm{C}$ e a $15^{\circ} \mathrm{C}$ pela Saccharomyces cerevisiae). As amostras fermentadas pela Kloeckera apiculata em relação a produção de álcool isoamílico apresentaram diferenças estatísticas tanto em relação ao mosto de uva, quanto em relação às temperaturas de fermentação.

Os ésteres são formados, principalmente durante a fermentação, da reação de álcoois e acetil-CoA, catalisada pela enzima álcool acetiltransferase. O etanol é o principal álcool do vinho e por isso, o acetato de etila, originado do etanol e do acetil-CoA, é o éster mais abundante do vinho [6]. A produção de acetato de etila foi maior nas amostras de mostos fermentados com a
Kloeckera apiculata, embora a produção deste composto nas amostras 1 e 2 (mostos de uvas fermentados com a Saccharomyces cerevisiae a $20^{\circ} \mathrm{C}$ ) não tenha apresentado diferenças significativas em relação as amostras 3' e 4' (mostos de uvas fermentados com a Kloeckera apiculata a $15^{\circ} \mathrm{C}$ ). ZOHRE \& ERTEN [19] também observaram alta produção de acetato de etila pela Kloeckera apiculata. A maior produção de acetato de isoamila aconteceu na amostra de mosto de uva Chardonnay fermentado a $20^{\circ} \mathrm{C}$ pela Kloeckera apiculata. Esta amostra diferiu estatisticamente de todas as outras amostras.

\section{4 - CONCLUSÕES}

As culturas puras de Kloeckera apiculata e Saccharomyces cerevisiae foram estudadas durante a fermentação de mosto de uva devido ao fato destas leveduras serem utilizadas em fermentações naturais, ou seja, espontâneas. A Kloeckera apiculata produziu quantidades significantes de compostos importantes para a qualidade do vinho como o acetato de etila, mas, por outro lado, a produção de etanol foi muito baixa, um ponto negativo para produção de vinho. Embora a produção de etanol pela Saccharomyces cerevisiae tenha sido alta, as produções de acetato de etila e acetato de isoamila foram baixas se comparadas com as amostras de mosto fermentado pela Kloeckera apiculata. O próximo passo deste trabalho será verificar a interação destas duas leveduras crescendo simultaneamente numa fermentação em um fermentador. A presença de leveduras que não são Saccharomyces cerevisiae durante a fermentação, em virtude da produção de compostos voláteis, pode ser de grande interesse para a aplicação biotecnológica da fabricação de vinho.

\section{5 - REFERÊNCIAS BIBLIOGRÁFICAS}

[1] BERRY, D.R. Alcoholic beverage fermentations. In: LEA, A.G.H.; PIGGOTT, J.R. Fermented beverage production, Blackie, London, p. 32-61, 1995.

[2] BILBAO, A.; IRASTORZA, A.; DUENAS, M.; FERNANDEZ, $\mathrm{K}$. The effect of temperature on the growth of strains of Kloeckera apiculata and Saccharomyces cerevisiae in apple juice fermentation. Lett. Appl. Microbiol. 24, p. 37-39, 1997.

[3] BISSON, L.F. Yeasts-Metabolism of sugars. In: FLEET, G. H. Wine Microbiology and Biotechnology, Harwood Academic Publishers, Chur, Switzerland, p. 55-68, 1994.

[4] BOULTON, R.B.; SINGLETON, V.I.; BISSON, I.F.; KUNKEE, R.E. Yeast and biochemistry of ethanol fermentation. In: Principles and Practices of Winemaking - Davis: Chapman \& Hall. p. 102-181, 1996.

[5] CIANI, M. Role, enological properties and potential use of non-Saccharomyces wine yeasts. Recent Res. Dev. Microbiol. 1, p. 317-331, 1997.

[6] DUFOUR, J.P.; MALCORPS, P. Esters synthesis during fermentation: enzyme characterization and modulation mechanism. In: CAMPBELL, I.; PRIEST, F. G. Proceedings of the fourth evermore conference on malting, The institute of brewing, London, p. 137-151, 1995. 
[7] ETIÉVANT, P.X. Wine. In: MAARSE, H. Volatile compounds in food and beverages, Marcel Dekker, New York, p. 483-546, 1991.

[8] FERREIRA, V.; LÓPEZ, R.; ESCUDERO, A.; CACHO, J. F. Quantitative determination of trace and ultratrace flavour active compounds in red wines through gas chromatographic-ion trap mass spectrometric analysis of microextracts. J. Chromatogr. 806, p. 349-354, 1998.

[9] FLEET, G.H.; HEARD, G.M. Growth during fermentation. In: FLEET, G.H. Wine microbiology and biotechnology, Harwood Academic Publishers, Chur, Switzerland, p. 27-54, 1994.

[10] GRANCHI, L.; GANUCCI, D.; MESSINI, A.; VINCENZINI, M. Oenological properties of Hanseniaspora osmophila and Kloeckera corticis from wines produced by spontaneous fermentations of normal and dried grapes. FEMS. 2, p. 403-407, 2002.

[11] HEARD, G.M.; FLEET, G.H. Occurence and growth of yeast species during the fermentation of some australian wines. Food Technol. Austr. 38, p. 22-25, 1986.

[12] HEARD, G.M.; FLEET, G.H. The effects of temperature and $\mathrm{pH}$ on the growth of yeast species during the fermentation of grape juice. J. Appl. Bacteriol. 65, p. 23-88, 1988.
[13] OUGH, C.S. Ammonia content of California grapes. Am. J. Enol. Vitic. 20, p. 213-220, 1969.

[14] OUGH, C.S.; AMERINE, M.A. Methods for analysis of musts and wines. New York. 377, 1988.

[15] PINNA, C.G.; HOGG, T.A. Microbial and chemical changes during the spontaneous ensilage of grape pomace. J. Appl. Microbiol. 86, p. 777-784, 1999.

[16] RIBEREAU, G.; PEYNAUD, P.J. Ácido sulfuroso. Analisis de vinos, Madrid: Aguilar. p. 310-322, 1958.

[17] ROMANO, P.; SUZZI, G. Sulfur dioxide and wine microorganisms. In: FLEET, G.H. Wine Microbiology and Biotechnology, Harwood Academic Publishers, Chur, Switzerland, p. 373-386, 1994.

[18] ROMANO, P.; SUZZI, G.; COMI, G.; ZIRONI, R. Higher alcohol and acetic acid production by apiculate wine yeasts. J. Appl. Bacteriol. 73, p. 126-130, 1992.

[19] ZOHRE, D.E.; ERTEN, H. The influence of Kloeckera apiculata and Candida pulcherrima yeasts on wine fermentation. Process Biochem. v. 38, n.3, p. 319324, 2002.

\section{6 - AGRADECIMENTOS}

As autoras agradecem o auxílio financeiro do CNPq e da Rede FINEP e a Chandon do Brasil S/A pela doação das amostras de mostos de uva Chardonnay e Pinot Noir. 